\title{
Spontaneous thoracic spinal cord herniation presenting as tethered cord syndrome
}

Sir,

A 36-year-old female presented with dysesthetic pain below the costal margin associated with progressive weakness of both lower limbs and wasting of right thigh muscles of one and half year duration and symptoms suggestive of upper motor neuron type of bladder of three months duration. Neurological examination revealed pyramidal signs in both the lower limbs, spasticity in the left lower limb and bilateral plantar extensor response. In addition she had wasting of right thigh muscles. Motor power was $4 / 5$ in the left lower limb and $0 / 5$ in the right lower limb. Magnetic resonance imaging (MRI) of the spine [Figure 1] revealed an anteriorly displaced cord at D4 level with obliteration of subarachnoid space and "S" shaped anterior kink of the spinal cord due to adherence of the cord to the posterior aspect of vertebral body through the dural defect. Patient underwent D3-D5 laminectomy and reduction of the herniation. At operation nerve roots were matted with

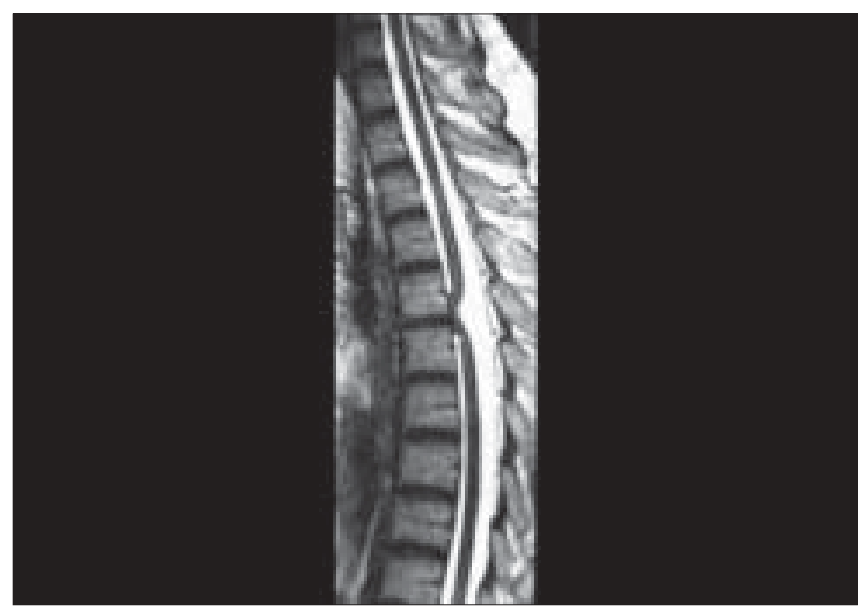

Figure 1: T2-weighted sagittal and axial MRI revealing an anteriorly displaced cord at D4 level with obliterated subarachnoid space anterior to it and S shaped anterior kink of spinal cord due to adherence of the cord to the posterior aspect of vertebral body through the dural defect s/o spinal cord herniation 


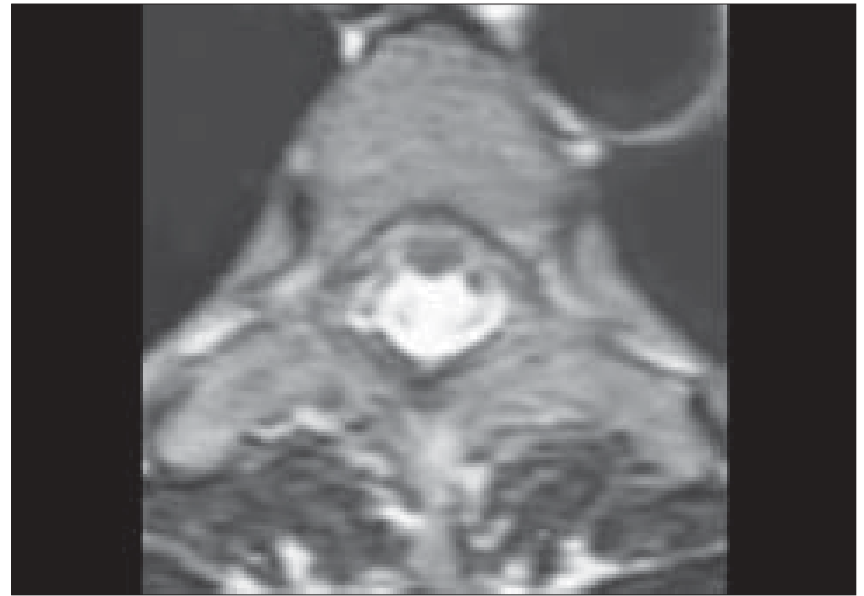

Figure 2: T2-weighted MRI spine axial view after surgical reduction showing well-reduced and straightened cord with no kink

thickened arachnoid. Cord was anteriorly adherent at D4 level. There was kinking and lateral rotation of the cord. Cord herniation through the small dural defect was identified and reduction was done by microsurgery and the defect was closed with teflon graft. Patient had good recovery and was able to ambulate with support. Postoperative MRI revealed good reduction of the herniation and restoration of normal anatomy [Figure 2].

Idiopathic anterior spinal cord herniation $(\mathrm{SCH})$ is a rare cause of thoracic myelopathy. ${ }^{[1,3-5]}$ Significant delay between the onset of symptoms and surgery is probably due to two factors: Rarity of the lesion and the entity often being misdiagnosed initially as posterior intradural arachnoid cyst. ${ }^{[2]}$ The mechanisms for the dural defect is still uncertain. The proposed mechanisms include: Minor trauma, duplication of the dura, rupture from prolapsed disc, congenital dural defect, and pressure erosion from the physiologically closely applied midthoracic cord to anterior dura. ${ }^{[2-4]}$ Regardless of the mechanism, progressive herniation of the cord occurs as a result of of cerebrospinal fluid pulsations as well as negative epidural pressure. ${ }^{[2,3]}$ This results in progressive neurological symptoms. In addition tethering of the cord restricts the normal movements of spinal cord associated with flexion of the spine ${ }^{[6]}$ The resultant traction of the cord may also be a contributory factor for the progressive neurological deficits. Other factors that may contribute to progressive myelopathy include distortion of the cord and cord ischemia. Though spontaneous spinal cord herniation is a very rare entity, early recognition and timely intervention can lead to good neurological recovery.

\section{Narayanam A. Sai Kiran, Sandeep Vaishya,} Manish K. Kasliwal, Shashank S. Kale, Bhavani S. Sharma

Department of Neurosurgery, All India Institute of Medical Sciences, New Delhi, India. E-mail:kalesaiaiims@yahoo.com

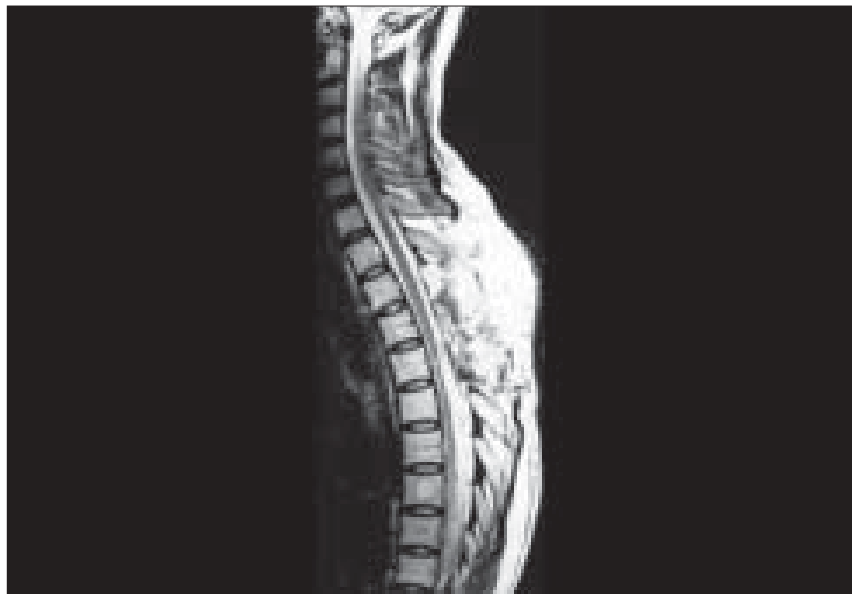

Figure 3: T2W sagittal MRI after surgery showing straightened cord with no kink

DOI: $10.4103 / 0028-3886.51306$

\section{References}

1. Barbagallo GM, Marshman LA, Hardwidge C, Gullan RW. Thoracic idiopathic spinal cord herniation at the vertebral body level: A sub group with poor prognosis. J Neurosurg 2002;97:369-74.

2. Tekkok HI. Spontaneous spinal cord herniation: Case report and review of literature. Neurosurgery 2000;46:485-92.

3. Worzman G, Tasker RR, Rewcastle NB, Richardson JC, Pearson FG. Spontaneous incarcerated herniation of the spinal cord into a vertebral body: A unique case of paraplegia. Case report. J Neurosurg 1974;41:631-5.

4. White BD, Tsegaye M. Idiopathic anterior spinal cord hernia: Under-recognized cause of thoracic myelopathy. Br J Neurosurg $2004 ; 18: 246-9$

5. Baur A, Stabler A, Psenner K, Hamburger C, Reiser M. Imaging findings in patients with ventral dural defects and herniation of neural tissue. Eur Radiol 1997;7:1259-63.

6. Adams RF, Anslow P. The natural history of transdural herniation of the spinal cord: Case report. Neuroradiology 2001;43:383-7.

Accepted on 12-03-2009 